\title{
Torsades de pointes observed in the early postoperative period in a patient with long QT syndrome
}

\author{
Min-Soo Kim ${ }^{1}$, Seung-Gyun Nam ${ }^{1}$, and Yong Seon Choi ${ }^{1,2}$ \\ ${ }^{1}$ Department of Anesthesiology and Pain Medicine, ${ }^{2}$ Anesthesia and Pain Research Institute, Yonsei University College of Medicine,
} Seoul, Korea

Long QT syndrome (LQTS) is an arrhythmogenic cardiac disorder that may occur congenitally as a result of mutations in genes encoding critical ion channels of the heart, metabolic abnormalities or drugs. Agents routinely used in general anesthesia, physical and emotional stress, enhanced sympathetic tone, and electrolyte imbalance during the perioperative period have been suggested to contribute to the development of torsades de pointes in patients with LQTS $[1,2]$. Therefore, in these patients, special attention and careful management are required to prevent fatal arrhythmia during the perioperative period.

A 56-year-old Asian female (height $169.4 \mathrm{~cm}$, weight 35 $\mathrm{kg}$ ) had undergone staging laparotomy due to ovarian cancer 20 months prior to presentation. At that time she presented with a prolonged QTc interval (QTc $=514 \mathrm{~ms}$ ) associated with syncope and a family history of unexplained sudden death of her brother, but the cardiac evaluation was mainly focused on assessing the presence of coronary artery occlusive disease since her father had angina. Cardiac evaluation including echocardiography, a treadmill test and computed tomography coronary angiography demonstrated no specific abnormal findings. Anesthesia was induced with $250 \mathrm{mg}$ thiopental and a $0.5 \mu \mathrm{g} / \mathrm{kg}$ bolus of remifentanil and was maintained with $0.1-$ $0.2 \mu \mathrm{g} / \mathrm{kg} / \mathrm{min}$ remifentanil and sevoflurane (1.5-2.0 vol\%). Approximately $3 \mathrm{hr}$ after surgery, cardiac arrest due to torsades de pointes developed suddenly.

Her electrocardiographic abnormalities were retrospectively reviewed. We found that the total LQTS score was 7; QTc interval
$>480$ ms: 3 , torsades de pointes: 2 , and syncope upon exertion or emotion: 2; hence she was diagnosed as having a high probability of LQTS in the absence of genetic testing [3]. Epinephrine provocation and isoproterenol tests were also positive. The patient was discharged on the 18th postoperative day with prescriptions for oral propranolol and potassium.

At the most recent presentation, the patient underwent elective segmental resection of the small bowel due to intestinal obstruction. Preoperative ECG showed T-wave inversion in leads V1-6 and a prolonged QT interval (QTc = $566 \mathrm{~ms})$. The patient was medicated with propranolol and potassium chloride until the morning of surgery. A defibrillator and all necessary antiarrhythmic drugs for the management of torsades de pointes were prepared before induction of anesthesia; propofol and remifentanil was administered with effect site concentrations of $4.0 \mu \mathrm{g} / \mathrm{ml}$ and $4.0 \mathrm{ng} / \mathrm{ml}$, respectively, using a target-controlled infusion device (Orchestra ${ }^{\mathrm{TM}}$, Fresenius Vial S.A, France). During operation, all electrolyte levels were maintained within normal ranges. There were no remarkable events during anesthesia maintenance. After extubation, the patient was transferred to the intensive care unit (ICU), where her vital signs were stable and pain scores measured on a visual analog scale (VAS) were between 20 and 50 .

Approximately seven hours after surgery, the patient complained of severe abdominal pain (VAS 80) and a blood pressure increased $(178 / 96 \mathrm{mmHg})$ with a heart rate of 79 beats/minute. She suddenly complained of palpitation and her ECG indicated ventricular bigeminy, ventricular tachycardia,

Corresponding author: Yong Seon Choi, M.D., Department of Anesthesiology and Pain Medicine, Anesthesia and Pain Research Institute, Yonsei University College of Medicine, 50, Yonsei-ro, Seodaemun-gu, Seoul 120-752, Korea. Tel: 82-2-2227-4576, Fax: 82-2-364-2951, E-mail: yschoi@yuhs.ac

(c) This is an open-access article distributed under the terms of the Creative Commons Attribution Non-Commercial License (http:// creativecommons.org/licenses/by-nc/3.0/), which permits unrestricted non-commercial use, distribution, and reproduction in any medium, provided the original work is properly cited. 


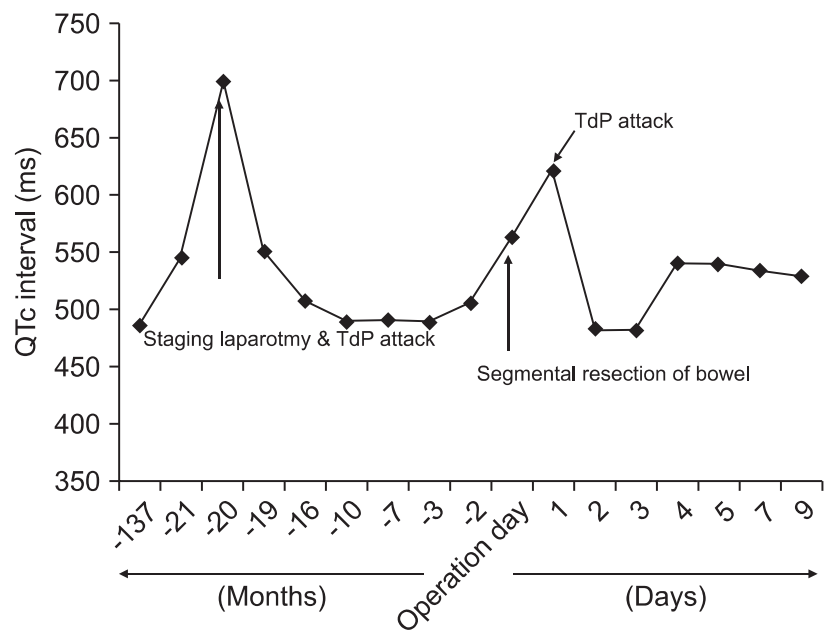

Fig. 1. Serial changes in the QTc interval during the perioperative period.

and torsades de pointes over a time frame of approximately 15 seconds. After checking her electrolyte levels $\left(\mathrm{K}^{+} 3.3 \mathrm{mmol} /\right.$ $\mathrm{L}, \mathrm{Ca}^{2+} 4.95 \mathrm{mg} / \mathrm{dl}$, and $\left.\mathrm{Mg}^{2+} 1.31 \mathrm{mg} / \mathrm{dl}\right), 20 \mathrm{mEq}$ of $\mathrm{KCl}$ was infused via the central venous catheter, and $2 \mathrm{~g}$ of magnesium sulfate was administered intravenously. Labetalol was started at a rate of $10 \mathrm{mg} / \mathrm{hr}$. The patient was discharged without any other events on the 11th postoperative day.

Patients with congenital LQTS may have an increased risk of developing malignant torsades de pointes in the perioperative period due to the influence of anesthetic drugs, surgical stress and postoperative pain on the QT interval. In present case, for induction and maintenance of anesthesia, thiopental, inhalation anesthetics, and atropine, which can prolong the QT interval, were avoided; propofol and remifentanil, which show no evidence of prolonging the QT interval, were administered using a target-controlled infusion device [4]. The ideal neuromuscular blocking agent should induce little or no histamine release and should not cause bradycardia, vagal stimulation and potassium shift. If possible, it should be short acting so that the use of reversal agents can be avoided because the use of anticholinesterase inhibitors with anticholinergics has been proposed to prolong the QT interval. We used rocuronium, which does not induce histamine release and has fewer autonomic effects. We did not use antiemetic agents due to the possibility of interaction between the 5-hydroxytryptamine 3 receptor antagonist and different human cardiac ion channels, thereby preventing QT interval prolongation [5]. We also assessed electrolyte levels regularly and corrected them quickly.

Interestingly, two different methods of anesthesia were performed in the same patient over a period of 20 months: volatile anesthetics using sevoflurane and total intravenous anesthesia using propofol and remifentanil before and after diagnosis of LQTS, respectively. However, regardless of anesthetic management, postoperative hypokalemia and increased sympathetic tone prolonged the QT interval and provoked torsades de pointes triggered by ventricular bigeminy. Episodes of torsades de pointes may be short-lived and self-terminating, but long bursts are a cause of severe hemodynamic compromise, and can degenerate into ventricular fibrillation [5]. In the episode of torsades de pointes that occurred after the first operation, the patient's hemodynamics were compromised and she required cardiopulmonary resuscitation. However, the episode of torsades de pointes following the second operation was short-lived and self-terminating. This may have been influenced by the difference in anesthetic management resulting from less prolongation of the QTc interval following the administration of total intravenous anesthesia compared to the volatile anesthetics used during the first operation (Fig. 1).

In conclusion, patients with LQTS require special attention and careful management throughout the perioperative period. A variety of agents used for general anesthesia can prolong the QT interval and lead to unexpected life-threatening dysarrhythmia. Patients with LQTS in the early postoperative period are still susceptible to torsades de pointes. Therefore, persistent monitoring of ECG and electrolytes, continued administration of a $\beta$-blocker and adequate postoperative pain control should be emphasized in the early postoperative period.

\section{References}

1. Booker PD, Whyte SD, Ladusans EJ. Long QT syndrome and anaesthesia. Br J Anaesth 2003; 90: 349-66.

2. Khan IA. Long QT syndrome: diagnosis and management. Am Heart J 2002; 143: 7-14.

3. Schwartz PJ, Moss AJ, Vincent GM, Crampton RS. Diagnostic criteria for the long QT syndrome. An update. Circulation 1993; 88: 782-4.

4. Kies SJ, Pabelick CM, Hurley HA, White RD, Ackerman MJ. Anesthesia for patients with congenital long QT syndrome. Anesthesiology 2005; 102: 204-10.

5. Kuryshev YA, Brown AM, Wang L, Benedict CR, Rampe D. Interactions of the 5-hydroxytryptamine 3 antagonist class of antiemetic drugs with human cardiac ion channels. J Pharmacol Exp Ther 2000; 295: 614-20. 